\title{
Analyse des dynamischen Verhaltens zweier gekoppelter Druckregelventile
}

\author{
Simon Schröders ${ }^{1}$ Alexander Fidlin ${ }^{1}$ \\ Eingegangen: 13. September 2019 / Angenommen: 7. März 2020 / Online publiziert: 18. März 2020 \\ (c) Der/die Autor(en) 2020
}

\section{Zusammenfassung}

In hydraulischen Systemen können Druckschwingungen aus unterschiedlichen Gründen und mit verschiedenen Amplituden und Frequenzen auftreten, zum Beispiel aufgrund von Pumpenpulsationen, hervorgerufen durch Instabilitäten hydraulischer Ventile oder durch externe Anregung. Die Reduktion dieser Druckschwingungen stellt somit bei der Konstruktion und Auslegung hydraulischer Systeme eine zentrale Herausforderung dar. Die gebräuchliche Lösung für diese Problematik besteht darin, hydraulische Widerstandselemente, welche die Schwingungen dämpfen sollen, zwischen verschiedenen Teilsystemen des hydraulischen Kreislaufs einzubringen. Dies ist energetisch ungünstig, da an jedem Widerstandselement Strömungs- und damit Leistungsverluste auftreten.

Dieser Beitrag behandelt die Modellbildung, Stabilitätsuntersuchung und dynamische Analyse eines Systems zweier gekoppelter Druckregelventile, wobei das erste Ventil als Druckbegrenzungsventil und das zweite als Druckminderventil fungiert. Das System stellt eine typische Konfiguration innerhalb eines hydraulischen Kreislaufs dar und beinhaltet die Problematik auftretender Druckschwingungen dadurch, dass die Gleichgewichtslagen der Ventile instabil werden können. Es wird gezeigt, dass bei dieser Ventilkonfiguration bei richtiger dynamischer Abstimmung die Ventilöffnung des Druckminderventils die Funktion einer sehr effizienten Dämpfungsblende erfüllen kann und in der Lage ist, Druckschwingungen, hervorgerufen durch das Druckbegrenzungsventil, signifikant zu reduzieren. Bei dieser Ventilkonfiguration kann somit auf zusätzliche Widerstandselemente verzichtet werden, weshalb sie energetisch besonders effizient ist.

Simon Schröders

simon.schroeders@kit.edu

Alexander Fidlin

alexander.fidlin@kit.edu
1 Institut für Technische Mechanik, Teilinstitut Dynamik/Mechatronik, Karlsruher Institut für Technologie, 76131 Karlsruhe, Deutschland 


\title{
Analysis of the oscillations in a system of two coupled pressure control valves
}

\begin{abstract}
In hydraulic systems, pressure oscillations can occur for different reasons and with different amplitudes and frequencies, for example due to pump pulsations, caused by instabilities of hydraulic valves or by external excitation. The reduction of these pressure oscillations thus represents a fundamental challenge in designing hydraulic systems. The most common solution to this problem is to introduce hydraulic resistance elements between different subsystems of the hydraulic circuit in order to damp the vibrations. This is energy inefficient, since power losses occur at each resistance element.

This contribution deals with the modelling, stability investigation and dynamic analysis of a system consisting of two coupled pressure control valves, namely a pressure relief valve and a pressure reducing valve. The system represents a typical configuration within a hydraulic circuit. It includes the problem of occurring pressure oscillations caused by possibly unstable equilibrium positions of the valves. Within this valve configuration, it is shown that, in the case of appropriate dynamical calibration, the valve opening of the pressure reducing valve can fulfil the function of a very efficient damping orifice and is capable of significantly reducing the pressure oscillations caused by the pressure relief valve. This valve configuration therefore eliminates the need for additional resistance elements and because of that can be particularly energy efficient.
\end{abstract}

\section{Einleitung}

In hydraulischen Systemen dienen Ventile als elementare Steuerelemente zur Regulierung der hydraulischen Leistungsübertragung. Da sie den Steuerungsaufwand minimieren, erfreuen sich selbstregelnde Druckventile großer Beliebtheit. Ihr Vorteil ist die Unabhängigkeit des zu regelnden Drucks sowohl von der aktuellen Versorgungs- als auch von der Verbrauchersituation. Außerdem können diese Ventile sowohl als Druckbegrenzungs- als auch als Druckminderventile betrieben, sowie sowohl als Schieberventile als auch als Sitzventile konstruiert werden. Ihr Nachteil ist jedoch ein möglicher Stabilitätsverlust, der durch die Kopplung zwischen hydraulischen und mechanischen Freiheitsgraden an Druckrückführflächen verursacht wird. Dies kann in bestimmten Betriebszuständen oder bei ungünstiger Parametrierung zum Auftreten von unerwünschten Schwingungen führen.

Aus dieser Problematik ergibt sich die Notwendigkeit für einen Kontrollmechanismus, der einerseits mögliche Druckschwingungen reduzieren kann und der andererseits, im Falle eines stabilen Betriebs, keine zusätzlichen Strömungsverluste erzeugt. In der Praxis werden meist hydraulische Widerstandselemente zwischen verschiedenen Teilsystemen des hydraulischen Kreislaufs eingebaut. Vor allem die gezielte Platzierung von Dämpfungsblenden sowie der Einsatz von Rückschlag- oder Volumenstrombegrenzungsventilen ist in diesem Zusammenhang üblich [2]. Dabei sollen die einzelnen Teilsysteme möglichst voneinander entkoppelt werden, um die Ausbreitung der Schwingungen in andere Teilsysteme zu verhindern. Der Nachteil dieser Strategie ist, dass sie aufgrund der Leistungsverluste an den hydraulischen Widerstandselementen energetisch ungünstig ist. Hier besteht Verbesserungspo- tential im Hinblick auf die Energiebilanz des hydraulischen Gesamtsystems.

Um neue Lösungen systematisch finden und untersuchen zu können, sind Stabilitätsanalysen und dynamische Untersuchungen der entsprechenden hydraulischen Systeme notwendig, da aufgrund komplexer nichtlinearer Systemzusammenhänge im Falle eines Stabilitätsverlustes das dynamische Verhalten meist schwierig vorherzusagen ist. Hierfür sind physikalische Modelle am geeignetsten, da mit ihnen Einblick in kausale Systemzusammenhänge und somit Systemverständnis gewonnen werden kann. Die physikalische Modellbildung hydraulischer Systeme ist in zahlreichen Standardwerken (siehe zum Beispiel [2, 6, 11]) ausführlich dokumentiert. Es gibt jedoch kaum Untersuchungen, welche diese Modelle hinsichtlich ihrer Stabilität und nichtlinearen Dynamik untersuchen. Untersuchungen in diesem Bereich fokussieren sich zudem aufgrund der interessanteren dynamischen Phänomene vor Allem auf Stoßschwingungen in Sitzventilen, siehe zum Beispiel [8]. Weiterhin existieren kaum Untersuchungen im Bereich der dynamischen Interaktion mehrerer Ventile.

Dieser Beitrag untersucht eine typische Systemkonfiguration bestehend aus einem Druckbegrenzungsventil und einem Druckminderventil. Die untersuchte Konfiguration ist eine mögliche Realisierung einer Widerstandssteuerung (auch Ventilsteuerung genannt) [1, 13], bei der die Steuerung der hydraulischen Leistung durch Drosselung des Volumenstroms an einem oder mehreren Ventilen erfolgt. Diese Art der hydraulischen Leistungssteuerung zeichnet sich dadurch aus, dass mit ihr hochdynamische und präzise Verstellungen des Verbraucherdrucks möglich sind. Nachteilig ist ein im Vergleich zu Verdrängersteuerungen schlechterer Wirkungsgrad, weshalb diese Art der Systemkonfiguration in Bereichen eingesetzt wird, in denen geringe Drücke und Volumenströme auftreten, aber schnelle und präzise Leis- 
tungsverstellungen erforderlich sind. Ein Anwendungsbeispiel ist die hydraulische Kupplungsbetätigung in einem Automatikgebriebe [3, 10, 12].

Zunächst werden beide Ventile separat betrachtet, physikalische Modelle hergeleitet und darauf basierende Stabilitätsanalysen durchgeführt. Nach der Synthese der beiden Ventile zu einem Gesamtsystem wird mit Hilfe numerischer Simulationen eine dynamische Analyse der Grenzzyklusschwingungen durchgeführt, welche auftreten, wenn das Druckbegrenzungsventil in den instabilen Betriebszustand gelangt. Systematische Parameterstudien zeigen auf, wie beide Ventile aufeinander abgestimmt werden müssen, damit die Schwingungsausbreitung durch das Druckminderventil möglichst reduziert und somit eine effiziente Schwingungsreduktion erzielt wird.

\section{Druckbegrenzungsventil}

Das vorliegende Kapitel beschreibt die Funktionsweise hydraulischer Druckbegrenzungsventile. Des weiteren wird die Herleitung eines mathematischen Modells auf Basis physikalischer Gesetzmäßigkeiten und Konstitutionsgleichungen diskutiert. Auf Basis dieses Modells wird eine Stabilitätsuntersuchung durchgeführt und kritische Parameter identifiziert.

\subsection{Funktionsprinzip}

Die Funktionsweise hydraulischer Druckbegrenzungsventile besteht darin, einen gewünschten Solldruck $p_{1}=p_{d}$ un-

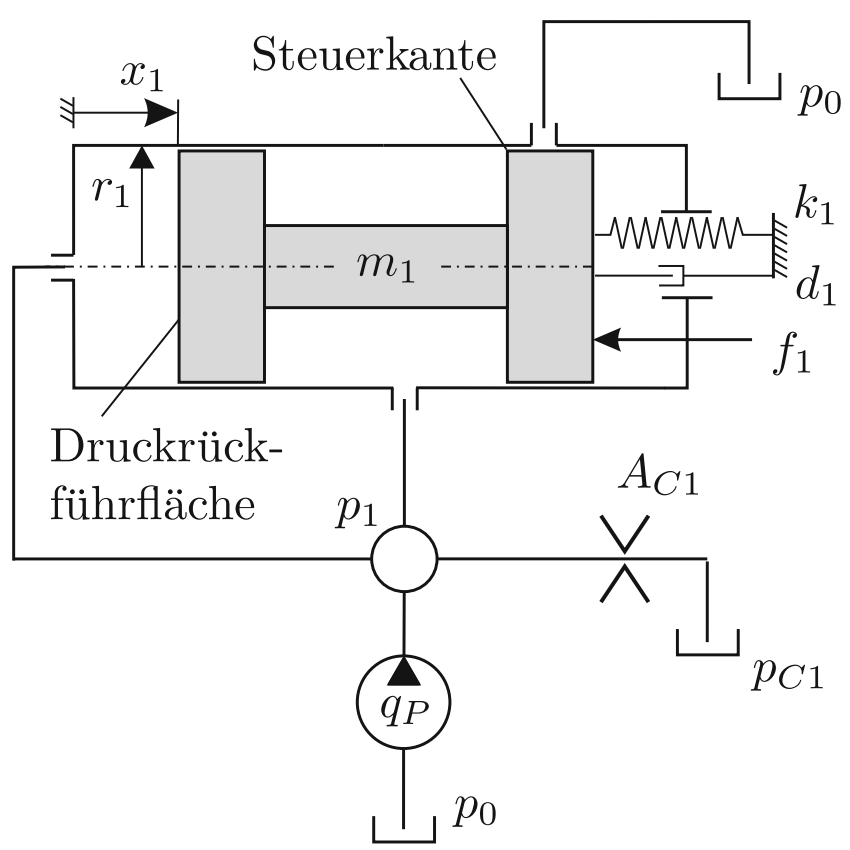

Abb. 1 Druckbegrenzungsventil abhängig von der aktuellen Versorgungs- (Volumenstrom $q_{P}$ ) und Verbrauchersituation (Verbraucherdruck $p_{C 1}$ beziehungsweise Blendenfläche $A_{C 1}$ ) aufrechtzuerhalten, vergleiche Abb. 1. Dies wird mit Hilfe der Kopplung zwischen mechanischen und hydraulischen Freiheitsgraden an der Druckrückführfläche realisiert. Aufgrund dieser Kopplung reagiert das Ventil auf Änderungen im Systemdruck $p_{1}$. Ist dieser zu niedrig, verringert das die Druckkraft auf den Ventilkolben, dieser verschiebt sich nach links und die Steuerkante schließt das Ventil. Dadurch kann sich der Druck neu aufbauen. Ist der Druck zu hoch, verschiebt sich aufgrund der größeren Druckkraft der Kolben nach rechts, wodurch mehr Volumenstrom durch die Ventilöffnung in den Tank fließen kann und der Systemdruck abnimmt. Der gewünschte Solldruck $p_{d}$ kann über die Federvorspannung oder einen Steuereingang $f_{1}$ stufenlos eingestellt sowie über das statische Kräftegleichgewicht am Kolben berechnet werden. Dieses Funktionsprinzip verläuft absolut passiv und erfordert keine aktive Regelung.

Es wird in Abb. 2 verdeutlicht, in der die Zeitverläufe der Kolbenposition $x_{1}$ und des Systemdrucks $p_{1}$ basierend auf einer numerischen Zeitintegration dargestellt sind. Auf eine Änderung der Blendenfläche $A_{C 1}$ zum Verbraucher (bei $t=t_{1}$ ) als auch auf eine Änderung des Pumpenvolumenstrom $q_{P}$ (bei $t=t_{2}$ ) antwortet das Ventil nach einem kurzen Einschwingvorgang stets mit dem gleichen Systemdruck $p_{1}$. Der Überschuss zwischen Versorgung und Verbraucher wird durch eine entsprechende Ventilposition, die den Volumenstrom über die Steuerkante des Ventils anpasst, automatisch kompensiert. Die in Kap. 1 erwähnte Stabilitätsproblematik wird durch eine Verringerung der Ventildämpfung $d_{1}$ (bei $t=t_{3}$ ) veranschaulicht, welche zu einer Destabilisierung der Gleichgewichtslage und zu daraus resultierenden selbsterregten Schwingungen führt.

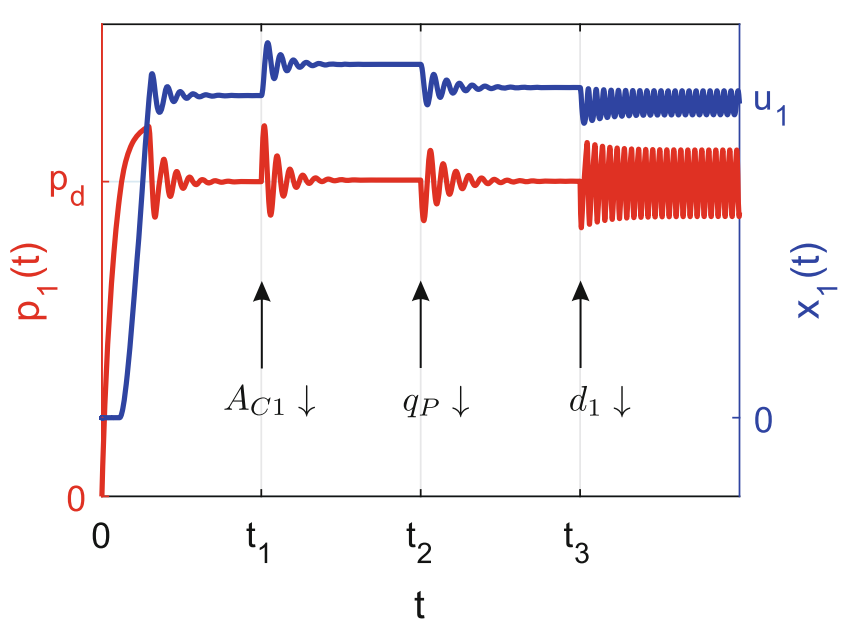

Abb. 2 Zeitverlauf von Kolbenverschiebung $x_{1}$ und Systemdruck $p_{1}$ 


\subsection{Modellbildung}

Bei der physikalischen Modellbildung jeglicher dynamischer Systeme muss stets ein Kompromiss zwischen Modellgüte und Rechenzeiteffizienz getroffen werden. Diesbezüglich wird beispielsweise in [7] aufgezeigt, dass das Stabilitätsproblem bei hydraulischen Druckbegrenzungsventilen bereits mit sehr einfachen Modellen beschrieben werden kann, bei denen Reibungseinflüsse, hydraulische Induktivitäten, laminare Rohrwiderstände und Strömungskräfte auf Ventilkolben vernachlässigt werden. Weiterhin zeigen Untersuchungen aus dem Bereich der Regelungstechnik [4, 5, 9], dass mit dieser Modellierungstiefe die entsprechenden Systemdynamiken sowohl qualitativ als auch quantitativ abgebildet werden können. Diesem Modellierungsansatz folgend, erhält man die Systemgleichungen

$m_{1} \ddot{x}_{1}+d_{1} \dot{x}_{1}+k_{1} x_{1}=r_{1}^{2} \pi p_{1}-f_{1}$,

$C_{h 1} \dot{p}_{1}=q_{P}-q_{V 1}-q_{C 1}-r_{1}^{2} \pi \dot{x}_{1}$

aus Kräftebilanzen an den Ventilkolben und aus Volumenstrombilanzen an den Kapazitäten. Dabei sind

$$
\begin{aligned}
& q_{V 1}=\gamma_{V} \sigma\left(x_{1}-u_{1}\right) 2 r_{1} \pi\left(x_{1}-u_{1}\right) \Delta\left(p_{1}-p_{0}\right), \\
& q_{C 1}=\gamma_{V} A_{C 1} \Delta\left(p_{1}-p_{C 1}\right)
\end{aligned}
$$

turbulente Steuerkanten- und Verbrauchervolumenströme und $\sigma(x)$ und $\Delta(p)$ sind definiert als

$\sigma(x)=\left\{\begin{array}{ll}1, & x \geq 0 \\ 0, & x<0\end{array}, \quad \Delta(p)=\left\{\begin{array}{ll}\sqrt{p}, & p \geq 0 \\ -\sqrt{|p|}, & p<0\end{array}\right.\right.$.

Weiterhin bezeichnen $m_{1}, d_{1}, k_{1}$ und $r_{1}$ die Masse, Dämpfung, Steifigkeit und den Radius des Ventilkolbens, $x_{1}$ ist die Kolbenverschiebung, $u_{1}$ die Ventilüberdeckung und $f_{1}$ der Steuereingang. Der Parameter $A_{C 1}$ ist die Blendenfläche zum Verbraucher, $C_{h 1}$ die hydraulische Kapazität, $\gamma_{\mathrm{V}}$ der turbulente Widerstandskoeffizient und $q_{P}$ ist der (ideale) Pumpenvolumenstrom. Die Drücke $p_{0}$ und $p_{C 1}$ bezeichnen Tankdruck und Druck im (idealen) Verbraucher. Ideal bedeutet in diesem Zusammenhang, dass sowohl Verbraucherdruck als auch Pumpenvolumenstrom als zeitlich kon- stant angenommen werden. Nach Einführung dimensionsloser Größen

$$
\begin{aligned}
& X_{i}(\tau)=\frac{x_{i}(t)-u_{i}}{u_{i}}, \quad P_{j}(\tau)=\frac{\left(p_{j}(t)-p_{0}\right) r_{i}^{2} \pi}{u_{i} k_{1}} \mu_{i}, \\
& \tau=\sqrt{\frac{k_{1}}{m_{1}}} t, \quad P_{S}=\frac{\left(p_{S}-p_{0}\right) r_{2}^{2} \pi \mu_{2}}{u_{2} k_{1}}, \quad D_{i}=\frac{1}{2} \frac{d_{i} \sqrt{\mu_{i}}}{\sqrt{m_{i} k_{1}}}, \\
& F_{i}=\frac{f_{i} \pm k_{i} u_{i}}{k_{1} u_{i}} \mu_{i}, \quad Q_{P}=\frac{r_{1}^{2} \pi \sqrt{m_{1}} q_{P}}{C_{h 1} k_{1}^{3 / 2} u_{1}}, \quad \kappa=\frac{u_{2 T}}{u_{2}}-1, \\
& \alpha_{i}=\frac{\sqrt{m_{i} u_{i}} 2 r_{i}^{2} \pi^{3 / 2} \gamma_{V}}{k_{1} C_{h i}} \mu_{i}, \quad \beta_{i}=\frac{r_{i}^{4} \pi^{2} \mu_{i}}{k_{1} C_{h i}}, \quad \lambda_{1}=\frac{k_{2}}{k_{1}} \mu_{2}, \\
& \gamma_{i}=\frac{A_{C i} \alpha_{i}}{u_{i} 2 r_{i} \pi}, \quad \lambda_{2}=\frac{r_{1} u_{2}}{r_{2} u_{1} \mu_{2}}, \quad \lambda_{3}=\frac{r_{2} u_{2}}{r_{1} u_{1}} \alpha_{1}
\end{aligned}
$$

mit $i=1,2, j=i, C i$ und $\mu_{1}=1, \mu_{2}=m_{1} / m_{2}$, lauten die dimensionslosen Systemgleichungen

$$
\begin{aligned}
X_{1}^{\prime \prime}+ & 2 D_{1} X_{1}^{\prime}+X_{1}=P_{1}-F_{1}, \\
P_{1}^{\prime}= & -\alpha_{1} \sigma\left(X_{1}\right) X_{1} \Delta\left(P_{1}\right)-\gamma_{1} \Delta\left(P_{1}-P_{C 1}\right) \\
& -\beta_{1} X_{1}^{\prime}+Q_{P} .
\end{aligned}
$$

Dabei bezeichnet ()$^{\prime}:=\frac{\mathrm{d}}{\mathrm{d} \tau}$ die Ableitung nach der dimensionslosen Zeit $\tau$. Die Größen für $i=2$ sowie $\lambda_{1}, \lambda_{2}, \lambda_{3}$, $P_{S}$ und $\kappa$ werden erst in Abschn. 3.2 benötigt.

\subsection{Stabilität der Gleichgewichtslage}

Im Folgenden wird eine Stabilitätsanalyse des Ventils durchgeführt. Aus den Gleichgewichtsbedingungen $X_{1}^{\prime \prime}=$ $0, X_{1}^{\prime}=0, P_{1}^{\prime}=0$ erhält man mit den dimensionslosen Systemgleichungen $(3,4)$ eine für den Sonderfall $P_{C 1}=0$ analytisch lösbare kubische Gleichung in $\sqrt{P_{1}^{*}}$, wobei $P_{1}^{*}$ den dimensionslosen Druck in der Gleichgewichtslage bezeichnet. Diese ergibt sich zu

$X_{1}^{*}=-F_{1}+P_{1}^{*}$,

$P_{1}^{*}=\frac{4}{3} \Lambda_{0} \cos \left(\frac{1}{3} \arccos \left(\frac{3}{2} \frac{Q_{P}}{\alpha_{1}} \sqrt{\frac{3}{\Lambda_{0}^{3}}}\right)\right)^{2}$

mit der Abkürzung $\Lambda_{0}=F_{1}-\gamma_{1} / \alpha_{1}$. Hierbei wurde der Fall $X_{1}^{*}>0$ vorausgesetzt, was bedeutet, dass das Druckbegrenzungsventil im stationären Betrieb geöffnet ist. Der Pumpenvolumenstrom muss dafür ausreichend groß sein, um den Verbrauchervolumenstrom zu kompensieren. Die dimensionslosen Systemgleichungen (4) werden nun um die Gleichgewichtslage linearisiert. Mit Hilfe der linearisierten Gleichungen können Aussagen über die Stabilität der Gleichgewichtslage des nichtlinearen Systems getroffen werden, falls die Gleichgewichtslage ein hyperbolischer Fixpunkt ist. Die Stabilitätsaussage erfolgt dabei anhand der Eigenwerte mit Hilfe des Hurwitz-Kriteriums. 


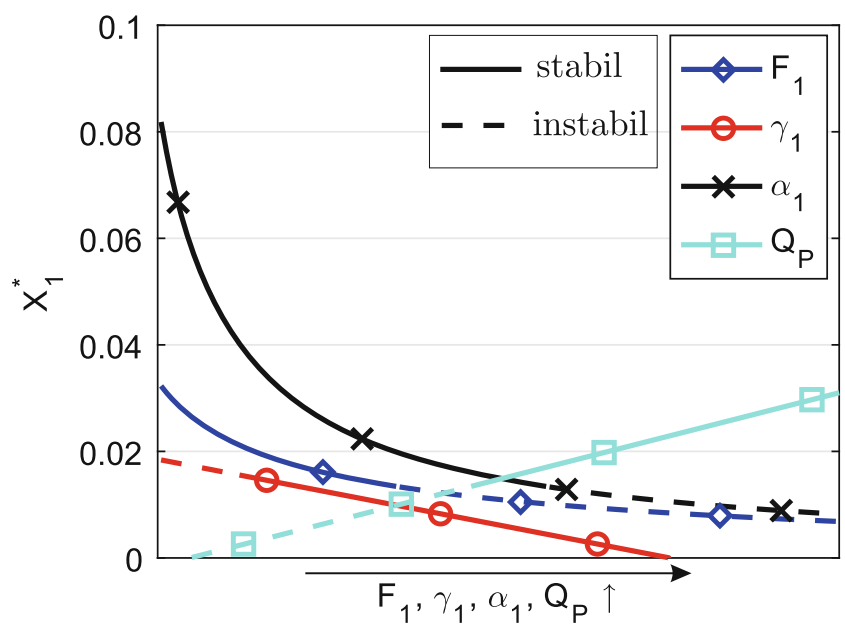

Abb. 3 Gleichgewichtslage und deren Stabilität. Dimensionslose Kolbenverschiebung $X_{1}^{*}$

In den Abb. 3 und 4 sind die Kolbenposition $X_{1}^{*}$ und der Druck $P_{1}^{*}$ in der Gleichgewichtslage sowie deren Stabilität in Abhängigkeit der Parameter $F_{1}, \gamma_{1}, \alpha_{1}$ und $Q_{\mathrm{P}}$ dargestellt. Dabei bezeichnen durchgezogene Linien stabile und gestrichelte Linien instabile Gleichgewichtslagen. Hier bestätigt sich das in Abschn. 2.1 beschriebene Funktionsprinzip, nämlich die Unabhängigkeit des Systemdrucks sowohl von der aktuellen Last (Parameter $\gamma_{1}$ ) als auch von der aktuellen Versorgung (dimensionsloser Volumenstrom $Q_{P}$ ), solange dieser ausreicht, den Verbrauchervolumenstrom zu kompensieren. Es zeigt sich, dass eine höhere dimensionslose Kraft $F_{1}$ und ein damit einhergehender höherer stationärer Systemdruck $P_{1}^{*}$ die Gleichgewichtslage destabilisieren kann. Die gleiche Tendenz gilt für den Parameter $\alpha_{1}$, welcher die Durchlassfläche durch das Druckbegrenzungsventil bestimmt. Einen gegenteiligen Einfluss zeigen der dimensionslose Pumpenvolumenstrom $Q_{P}$ und die dimensionslose Blendenfläche zum Verbraucher $\gamma_{1}$. Im vorliegenden Fall wird bei Erhöhung dieser Parameterwerte die Gleichgewichtslage stabilisiert.

\section{Druckminderventil}

Das vorliegende Kapitel ist analog zu Kap. 2 aufgebaut und diskutiert das Funktionsprinzip, die Modellbildung und die Stabilitätsanalyse von Druckminderventilen.

\subsection{Funktionsprinzip}

Ebenso wie beim Druckbegrenzungsventil (vergleiche Kap. 2) besteht das Funktionsprinzip von Druckminderventilen darin, dass über das Gleichgewicht zwischen einem Steuereingang $f_{2}$ und der über die Kolbenfläche induzierten Druckkraft der Systemdruck $p_{2}$ stufenlos ein-

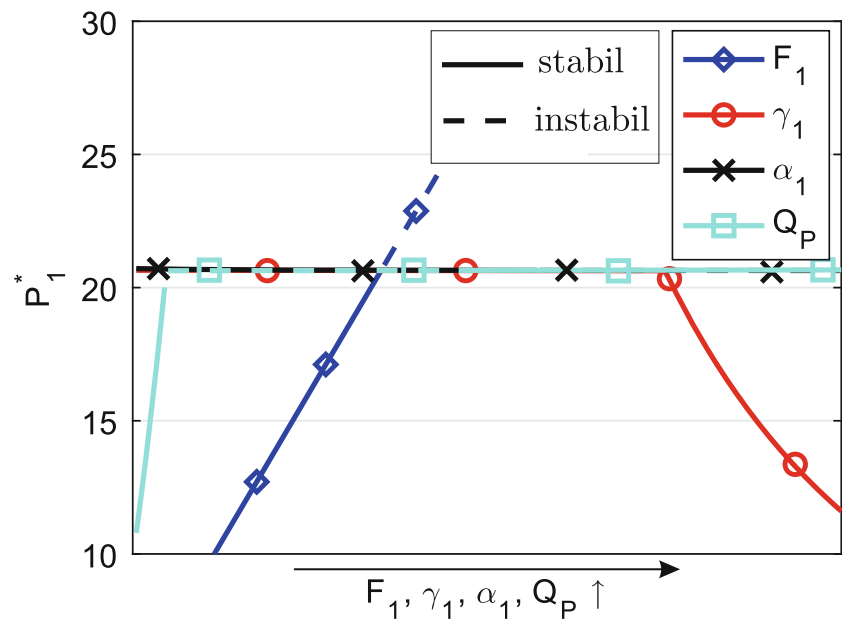

Abb.4 Gleichgewichtslage und deren Stabilität. Dimensionsloser Systemdruck $P_{1}^{*}$

gestellt werden kann. Die Konfiguration als Druckminderventil benötigt eine zusätzliche Ventilöffnung, an den die Druckquelle angeschlossen wird, vergleiche Abb. 5. Diese Konfiguration mit zwei Ventilöffnungen ermöglicht die Befüllung und vollständige Entleerung der Systemkapazität, weshalb sich diese Konfiguration als Aktuationsventil für einen Verbraucher anbietet.

\subsection{Modellbildung}

Dem Modellierungsansatz aus Abschn. 2.2 folgend, ergeben sich die Systemgleichungen des Druckminderventils zu

$m_{2} \ddot{x}_{2}+d_{2} \dot{x}_{2}+k_{2} x_{2}=-r_{2}^{2} \pi p_{2}+f_{2}$,
$C_{h 2} \dot{p}_{2}=q_{V 2_{S}}-q_{V 2_{T}}-q_{C 2}+r_{2}^{2} \pi \dot{x}_{2}$

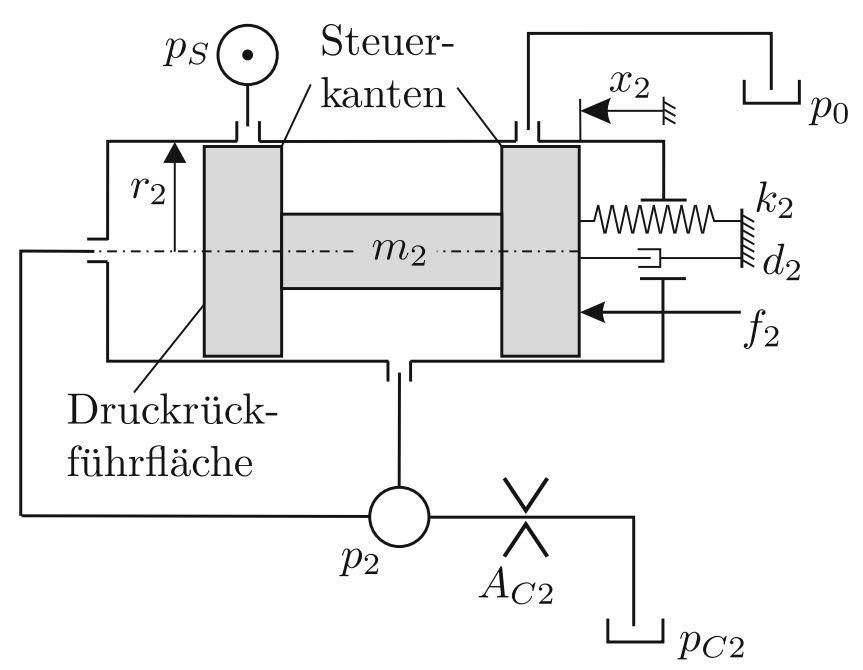

Abb. 5 Druckminderventil 


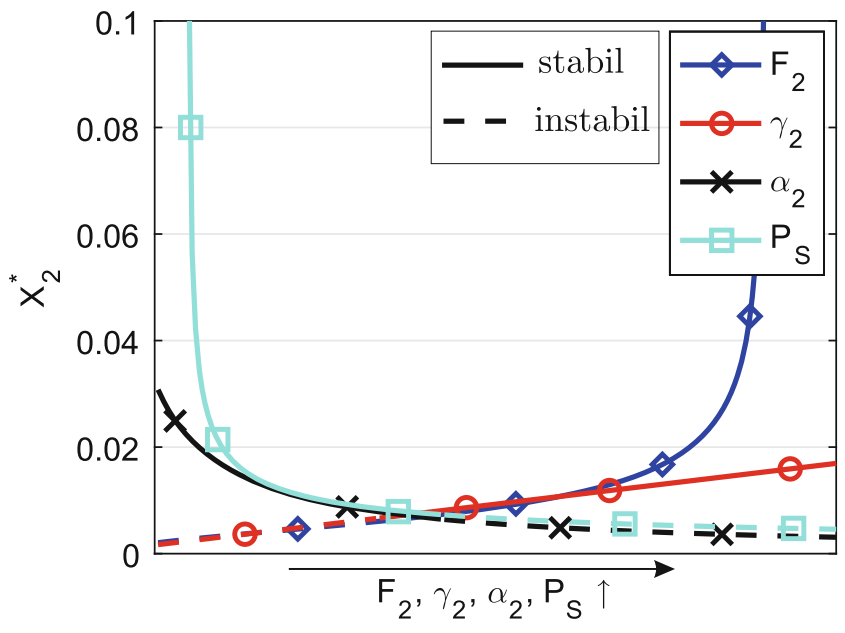

Abb. 6 Gleichgewichtslage und deren Stabilität. Dimensionslose Kolbenverschiebung $X_{2}^{*}$

mit den turbulenten Volumenströmen

$$
\begin{aligned}
& q_{C 2}=\gamma_{V} A_{C 2} \Delta\left(p_{2}-p_{C 2}\right), \\
& q_{V 2_{T}}= \gamma_{V} \sigma\left(-\left(x_{2}-u_{2 T}\right)\right) 2 r_{2} \pi\left(-\left(x_{2}-u_{2 T}\right)\right) \\
& \Delta\left(p_{2}-p_{0}\right), \\
& q_{V 2_{S}}=\gamma_{V} \sigma\left(x_{2}-u_{2}\right) 2 r_{2} \pi\left(x_{2}-u_{2}\right) \Delta\left(p_{S}-p_{2}\right) .
\end{aligned}
$$

Die Parameterbezeichnungen sind analog zu Abschn. 2.2 eingeführt, wobei für den vorliegenden Abschnitt der Index 2 verwendet wird. Weiterhin werden zusätzlich zum Druckbegrenzungsventil eine zweite Ventilüberdeckung $u_{2 T}$ sowie der Versorgungsdruck $p_{S}$ eingeführt. Mit den dimensionslosen Größen aus Abschn. 2.2 lauten die dimensionslosen Systemgleichungen

$$
\begin{aligned}
X_{2}^{\prime \prime}+ & 2 D_{2} X_{2}^{\prime}+\lambda_{1} X_{2}=-P_{2}+F_{2} \\
P_{2}^{\prime}= & \alpha_{2} \sigma\left(X_{2}\right) X_{2} \Delta\left(P_{S}-P_{2}\right)-\gamma_{2} \Delta\left(P_{2}-P_{C 2}\right) \\
& +\beta_{2} X_{2}^{\prime}-\sigma\left(\kappa-X_{2}\right)\left(\kappa-X_{2}\right) \alpha_{2} \Delta\left(P_{2}\right) .
\end{aligned}
$$

\subsection{Stabilität der Gleichgewichtslage}

Die Gleichgewichtslage des Druckminderventils lässt sich ebenso wie beim Druckbegrenzungsventil lediglich für den Sonderfall $P_{C 2}=0$ analytisch angeben. Nach Lösung einer kubischen Gleichung in $\sqrt{P_{2}^{*}}$ erhält man

$$
\begin{aligned}
& X_{2}^{*}=F_{2}-P_{2}^{*}, \\
& P_{2}^{*}=\frac{2}{3} F_{2}+\frac{1}{3} P_{S}-\frac{2}{3} \sqrt{\Lambda_{1}} \cos \left(\frac{1}{3} \arccos \left(\frac{\Lambda_{2}}{\sqrt{\Lambda_{1}^{3}}}\right)\right)
\end{aligned}
$$

mit den Abkürzungen $\Lambda_{1}=\left(F_{2}-P_{S}\right)^{2}-3 \frac{\gamma_{2}^{2}}{\alpha_{2}^{2}} \lambda_{1}^{2}$ und $\Lambda_{2}=$ $\left(F_{2}-P_{S}\right)^{3}+9 \frac{\gamma_{2}^{2}}{\alpha_{2}^{2}} \lambda_{1}^{2}\left(F_{2}+\frac{1}{2} P_{S}\right)$.

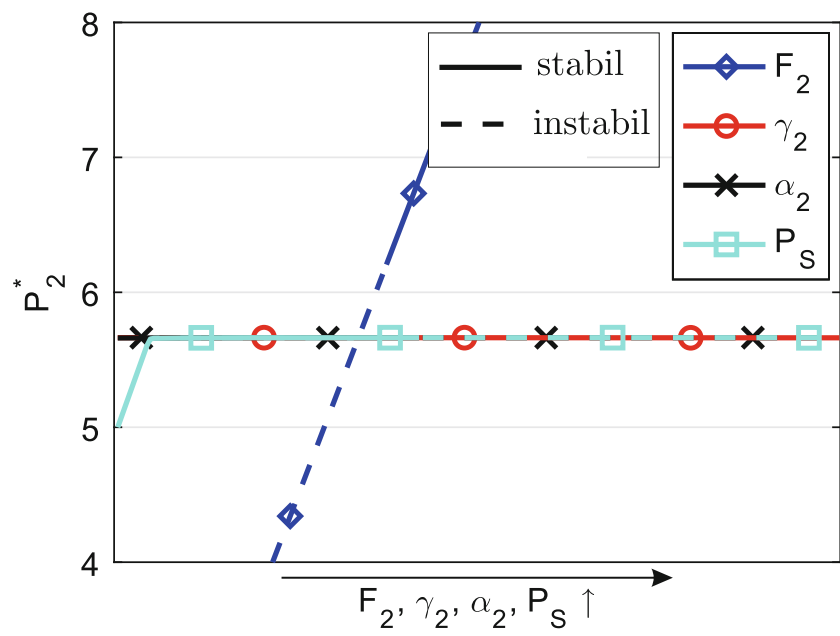

Abb.7 Gleichgewichtslage und deren Stabilität. Dimensionsloser Systemdruck $P_{2}^{*}$

Abb. 6 und 7 zeigen die Kolbenposition $X_{2}^{*}$ und den Druck $P_{2}^{*}$ in der Gleichgewichtslage, sowie die Stabilität der Gleichgewichtslage in Abhängigkeit der Parameter $F_{2}$, $\gamma_{2}, \alpha_{2}$ und $P_{S}$. Man erkennt auch beim Druckminderventil die Unabhängigkeit des Systemdrucks $P_{2}^{*}$ sowohl vom Versorgungsdruck $P_{S}$ als auch vom Verbrauchervolumenstrom (beschrieben durch Parameter $\gamma_{2}$ ). Bezüglich der Stabilität wirkt im Gegensatz zum Druckbegrenzungsventil ein höherer Gleichgewichtsdruck $P_{2}^{*}$ hier stabilisierend. Die dimensionslosen Parameter $\alpha_{2}$ (Erhöhung des Parameters destabilisiert) und $\gamma_{2}$ (Erhöhung des Parameters stabilisiert) haben im Vergleich zum Druckbegrenzungsventil bezüglich der Stabilität qualitativ den gleichen Einfluss.

\section{System zweier gekoppelter Ventile}

Im vorliegenden Kapitel wird gezeigt, wie die beiden bisher gezeigten Ventilvarianten zu einer typischen Konfiguration innerhalb eines hydraulischen Kreislaufs verbunden werden. Weiterhin wird eine dynamische Analyse durchgeführt für den Fall, dass eines der Ventile in den instabilen Betriebszustand kommt. Gezielte Parameterstudien zeigen auf, wie die Ventile aufeinander abgestimmt werden müssen, sodass die Ausbreitung der Schwingungen effizient reduziert wird.

\subsection{Funktionsprinzip}

Eine typische Ventilkonfiguration innerhalb eines hydraulischen Kreislaufs besteht in der Hintereinanderschaltung eines Druckbegrenzungsventils und eines Druckminderventils, vergleiche Abb. 8. Ersteres Ventil dient der Regulierung beziehungsweise Aufrechterhaltung eines gewünschten Systemdrucks $p_{1}$. Das zweite Ventil kann durch Än- 


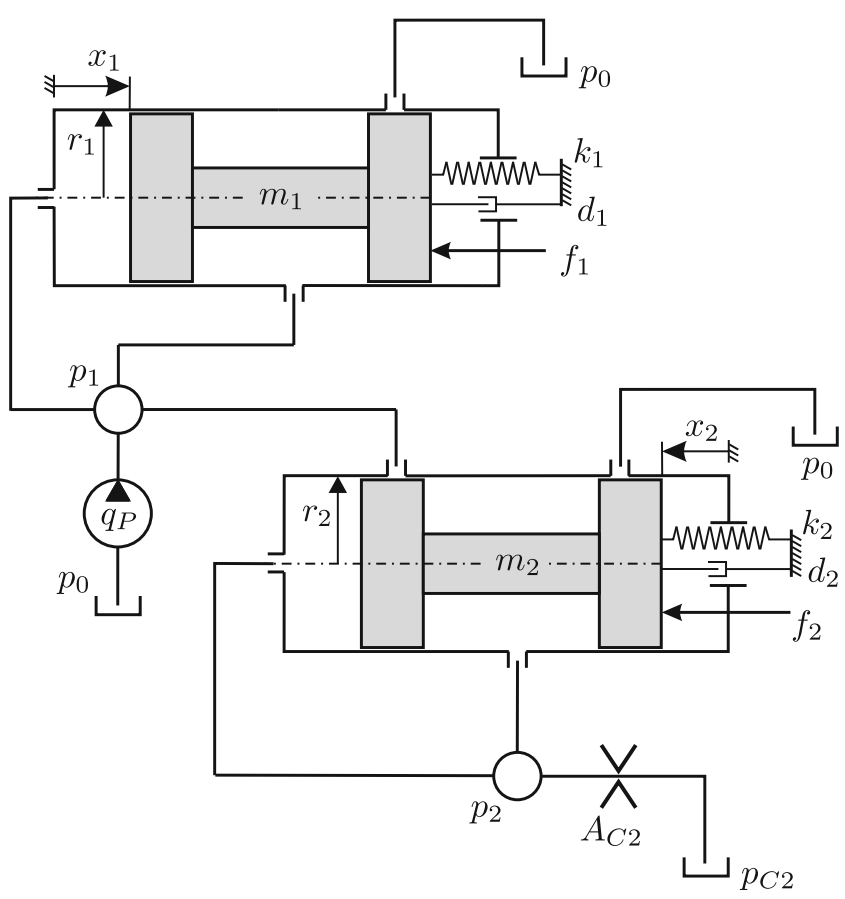

Abb. 8 System aus Druckbegrenzungsventil und Druckminderventil

derung des Drucks $p_{2}$ einen Verbraucher aktuieren. Das Druckbegrenzungsventil stellt in dieser Konfiguration also eine reale Druckquelle für das Druckminderventil dar.

Da der Druck $p_{2}$ durch Drosselung des Volumenstroms am Druckminderventil reguliert wird, handelt es sich bei der in Abb. 8 gezeigten Systemkonfiguration um eine Widerstandssteuerung. Diese Konfiguration ermöglicht eine sehr schnelle und präzise Verstellung des Verbraucherdrucks $p_{2}$, hat jedoch im Vergleich zu Systemen in Verdrängersteuerung einen wesentlich schlechteren Wirkungsgrad.

\subsection{Modellbildung}

Zur Bestimmung der Systemgleichungen muss in der Druckaufbaugleichung (2) des Druckbegrenzungsventils der Verbrauchervolumenstrom $q_{C 1}$ durch den Ventilvolumenstrom $q_{V 2_{S}}$ ersetzt werden. Weiterhin dient der Systemdruck $p_{1}(t)$ des Druckbegrenzungsventils nun als Versorgungsdruck $p_{S}$ für das Druckminderventil. Die dimensionslosen Gleichungen lauten entsprechend

$$
\begin{aligned}
X_{1}^{\prime \prime} & +2 D_{1} X_{1}^{\prime}+X_{1}=P_{1}-F_{1} \\
P_{1}^{\prime}= & -\alpha_{1} \sigma\left(X_{1}\right) X_{1} \Delta\left(P_{1}\right)-\beta_{1} X_{1}^{\prime} \\
& -\sigma\left(X_{2}\right) X_{2} \lambda_{3} \Delta\left(P_{1}-\lambda_{2} P_{2}\right)+Q_{P} \\
X_{2}^{\prime \prime} & +2 D_{2} X_{2}^{\prime}+\lambda_{1} X_{2}=-P_{2}+F_{2} \\
P_{2}^{\prime} & =\frac{\alpha_{2}}{\sqrt{\lambda_{2}}} \sigma\left(X_{2}\right) X_{2} \Delta\left(P_{1}-\lambda_{2} P_{2}\right)+\beta_{2} X_{2}^{\prime} \\
& -\gamma_{2} \Delta\left(P_{2}-P_{C 2}\right)-\sigma\left(\kappa-X_{2}\right)\left(\kappa-X_{2}\right) \alpha_{2} \Delta\left(P_{2}\right) .
\end{aligned}
$$

\subsection{Dynamische Analyse}

In den Abschn. 2.3 und 3.3 wurden die Stabilität beeinflussende Parameter identifiziert. Wird dies in der Konstruktion und Auslegung der Ventile berücksichtigt, kann, unter Kenntnis aller Systemparameter, ein stabiler Betrieb gewährleistet werden. Bei genauerer Analyse zeigt sich jedoch ein Zielkonflikt zwischen Stabilität und Schnelligkeit der Ventildynamik. Beispielsweise führt eine Vergrößerung der Ventildämpfung oder eine Verkleinerung des Kolbenradius' zwar zu einer Stabilisierung der Ventile, jedoch verschlechtern sich gleichzeitig die dynamischen Eigenschaften des Systems. Dies äußert sich zum Beispiel in einer längeren Einschwingzeit oder stärker ausgeprägtem Überschwingverhalten nach einer Veränderung des Betriebspunkts, wie sie beispielsweise in Abb. 2 zum Zeitpunkt $t=t_{2}$ gezeigt wird. Ebenso wird die Dynamik bei Änderungen des Steuersignals langsamer. Andere Parameter, wie der Steuereingang oder der Pumpenvolumenstrom sind nicht beliebig frei wählbar, da sie den stationären Systemdruck verändern oder Nebenbedingungen verletzen. Somit ist zumeist ein Betrieb möglichst nahe der Stabilitätsgrenze erwünscht, da hier die Reaktionszeit der Ventile am besten ist. Dies birgt die Gefahr, in den instabilen Betriebszustand zu gelangen, falls sich Parameter während des Betriebs ändern. Es muss somit eine Strategie entwickelt werden, wie möglicherweise auftretende Druckschwingungen reduziert werden können beziehungsweise wie deren Ausbreitung in andere Teilsysteme verhindert werden kann. Im Folgenden wird der Fall betrachtet, dass die Gleichgewichtslage des Druckbegrenzungsventils instabil wird, während das Druckminderventil im stabilen Bereich betrieben wird.

In den Abb. 9 und 10 sind für diesen Fall die Frequenzspektren der dimensionslosen Druckverläufe $P_{1}$ und $P_{2}$, bezogen auf die maximale Amplitude $A_{P_{1}}$ des Spektrums von $P_{1}$, für unterschiedliche Verbraucherströme (Parameter $\gamma_{2}$ ) dargestellt. Offensichtlich wirkt sich die Größe des Verbraucherstroms auf die Amplitude $A_{P_{1}}$ aus, siehe Abb. 9. Besonders auffällig ist jedoch, dass gegenüber der Amplitude $A_{P_{1}}$ eine signifikante Reduktion der Schwingungsamplitude $A_{P_{2}}$ um $99 \%$ bis $99,9 \%$ erreicht wird (Abb. 10). Dies ist dadurch zu erklären, dass die Ventilöffnungen die Wirkung von Blenden mit variabler Durchlassfläche haben. Blenden sind hydraulische Widerstandselemente, an denen die Energiedissipation proportional zum Quadrat des sie durchflieBenden Volumenstroms und somit umgekehrt proportional zum Quadrat ihrer Durchlassfläche ist. Die Energiedissipation ist also dann besonders hoch, wenn die Durchlassfläche klein ist. Beim Druckminderventil ist die Ventilöffnung zum Druckbegrenzungsventil dann klein, wenn $X_{2}^{*} \ll 1$, das heißt wenn das Ventil in der Gleichgewichtslage nur leicht geöffnet ist (Es gilt stets $X_{2}^{*}>0$, falls $\gamma_{2}>0$ ). Aus Abb. 6 geht hervor, dass die Ventilöffnung in der Gleich- 


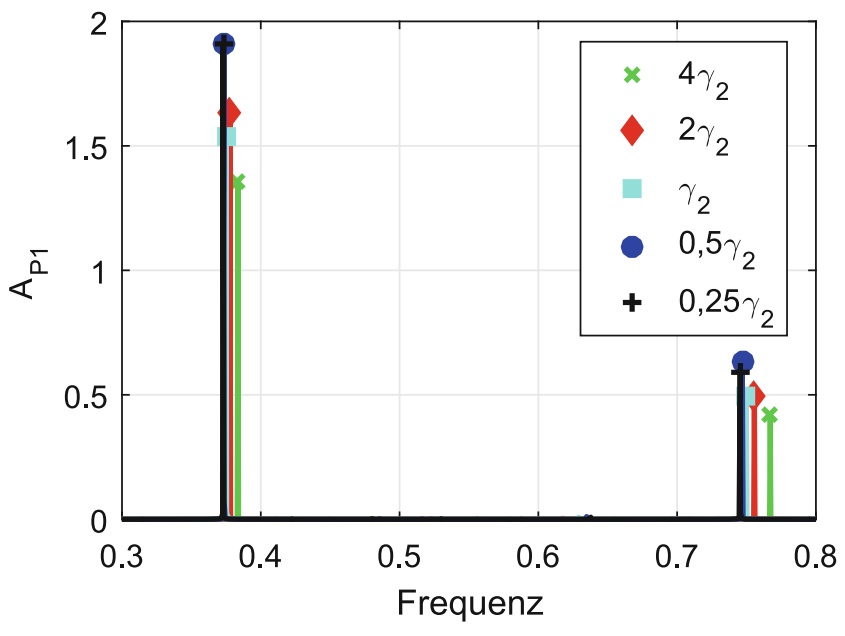

Abb. 9 Frequenzspektrum des dimensionslosen Systemdrucks $P_{1}$ in Abhängigkeit verschiedener Verbraucherströme (Parameter $\gamma_{2}$ )

gewichtslage proportional zum Verbraucherstrom (Parameter $\gamma_{2}$ ) ist, deshalb ist die Energiedissipation und somit die Dämpfungswirkung ebenfalls besonders hoch, wenn $\gamma_{2}$ klein ist. Dieser Effekt lässt sich somit besonders für solche Verbraucher ausnutzen, welche im stationären Betrieb nur wenig Volumenstrom benötigen, zum Beispiel für Arbeitszylinder, bei denen der Verbraucherstrom im stationären Betrieb einem Leckagestrom entspricht und somit besonders klein ist.

\subsection{Das fremderregte Druckminderventil}

Der vorherige Abschnitt hat gezeigt, dass Schwingungen, welche das Druckminderventil erreichen, durch dessen Steuerkante sehr effektiv gedämpft werden können. Im vorliegenden Abschnitt wird untersucht, ob dieser Effekt

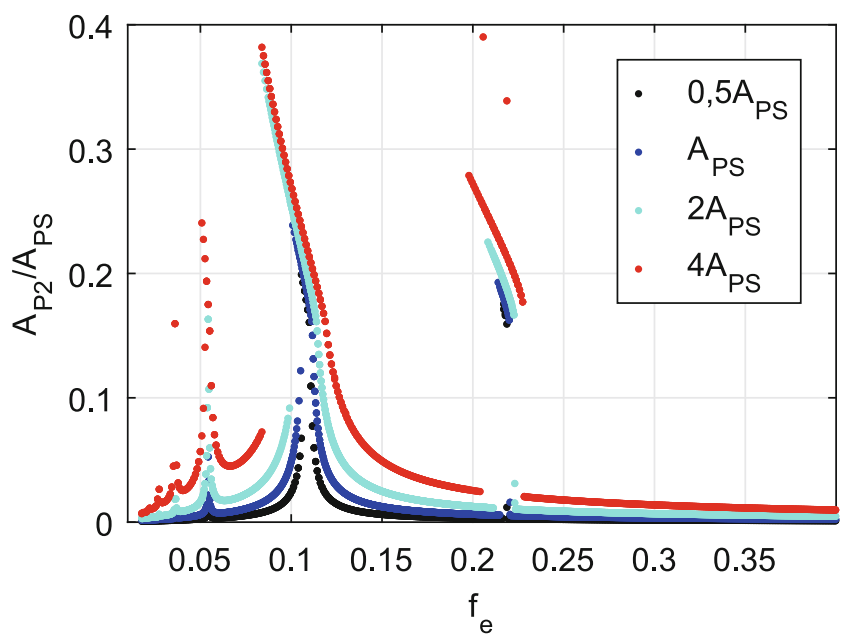

Abb. 11 Nichtlinearer Amplitudengang des Drucks $P_{2}$ bezogen auf die Anregungsamplitude $A_{P_{S}}$, wobei $A_{P_{2}}:=1 / 2\left(\max \left(P_{2}\right)-\right.$ $\min \left(P_{2}\right)$ ) bei Variation der Anregungsamplitude $A_{P_{S}}$

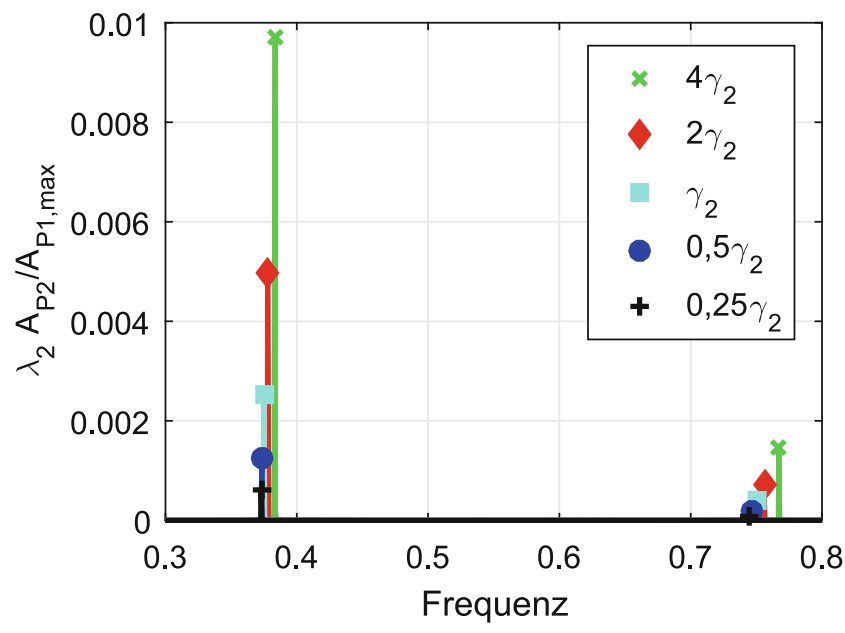

Abb. 10 Frequenzspektrum des dimensionslosen Druck $P_{2}$ bezogen auf $P_{1}$ in Abhängigkeit verschiedener Verbraucherströme (Parameter $\left.\gamma_{2}\right)$

über einen breiten Parameter- und Betriebsbereich genutzt werden kann und wie die Ventile aufeinander abgestimmt werden müssen, um den Dämpfungseffekt optimal zu nutzen. Dafür werden im Folgenden die selbsterregten Schwingungen des Druckbegrenzungsventils als harmonische Anregung durch den dimensionslosen Versorgungsdruck

$P_{S}=P_{S}(\tau)=P_{1}^{*}+A_{P_{S}} \cos \left(2 \pi f_{e} \tau\right)$

modelliert, wobei $P_{1}^{*}$ dem dimensionslosen Gleichgewichtsdruck des Druckbegrenzungsventils entsprechend Gl. (6) entspricht.

Abb. 11 zeigt den auf die Anregungsamplitude $A_{P_{\mathrm{S}}}$ bezogenen nichtlinearen Amplitudengang des Drucks $P_{2}$

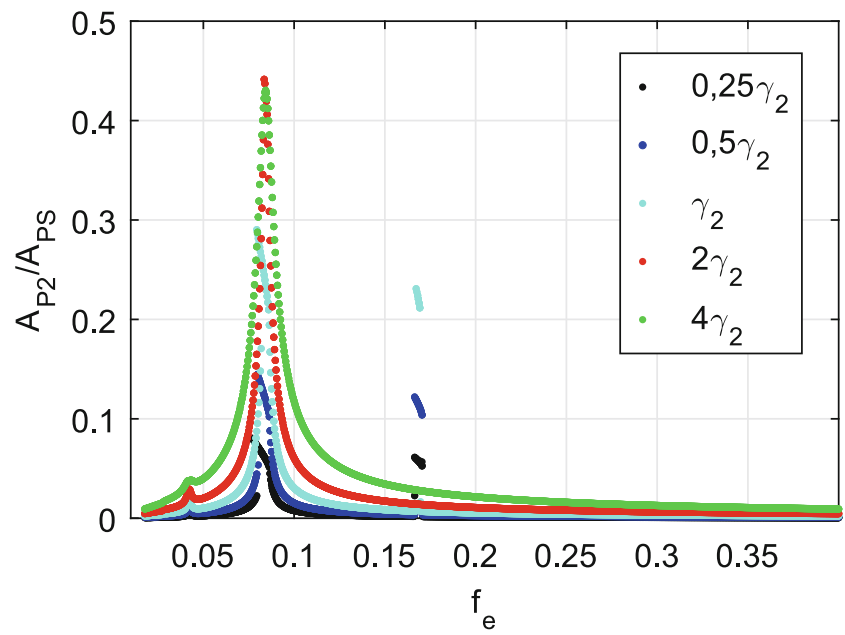

Abb. 12 Nichtlinearer Amplitudengang des Drucks $P_{2}$ bezogen auf die Anregungsamplitude $A_{P_{S}}$, wobei $A_{P_{2}}:=1 / 2\left(\max \left(P_{2}\right)-\right.$ $\min \left(P_{2}\right)$ ) bei Variation des Verbraucherstroms (Parameter $\left.\gamma_{2}\right)$ 
für verschiedene Anregungsamplituden $A_{P_{S}}$. Zu sehen ist eine nichtlineare Resonanz bei $f_{e} \approx 0,1$ sowie mehrere sub- und superharmonische Resonanzen. In Abb. 12 ist der entsprechende Amplitudengang für verschiedene Verbraucherströme (Parameter $\gamma_{2}$ ) dargestellt. Wie schon in Abschn. 4.3 angedeutet, wird die Dämpfungswirkung schlechter, je mehr Volumenstrom der Verbraucher benötigt. Trotzdem kann auch hier bei richtiger Abstimmung der Schwingungsfrequenz eine sehr gute Dämpfung erzielt werden. Falls die Anregung in einer der Resonanzfrequenzen vermieden wird, kann also insgesamt eine sehr gute Schwingungsreduktion erreicht werden. Wie die Frequenz der selbsterregten Schwingungen des Druckbegrenzungsventils beeinflusst werden kann, bedarf weiterer Untersuchungen, beispielsweise in Form numerischer Simulationen.

\section{Zusammenfassung und Ausblick}

Im Rahmen dieses Beitrags wurde die Modellbildung, Stabilitätsuntersuchung und dynamische Analyse hydraulischer selbstregelnder Ventile untersucht. Dabei wurden zwei Ventiltypen betrachtet, wobei sich das Druckbegrenzungsventil zur Druckregelung und das Druckminderventil zur Aktuierung eines Verbrauchers eignet. Nachdem für die Stabilität kritische Parameter mit Hilfe einer Stabilitätsuntersuchung identifiziert wurden, wurde gezeigt, dass eine Manipulation dieser Parameter zu einem Zielkonflikt hinsichtlich der Reaktionszeit der Ventile führt. Eine dynamische Analyse des Systems aus zwei gekoppelten Ventilen zeigte auf, dass die Steuerkante des Druckminderventils als sehr effektive Dämpfungsblende agieren kann. Um diesen Effekt optimal auszunutzen zu können, müssen die Ventile dynamisch so aufeinander abgestimmt werden, dass keine Resonanzfrequenz getroffen wird. Weiterhin lässt sich dieser Effekt besonders für solche Systeme ausnutzen, bei denen im stationären Betrieb der Verbraucherstrom lediglich einem Leckagestrom entspricht. Abschließend lässt sich sagen, dass diese Herangehensweise dem Einsatz zusätzlicher hydraulischer Widerstandselemente überlegen ist und das Potential hat, die Energieeffizienz des Gesamtsystems zu steigern. Wie die Frequenz der selbsterregten Schwingungen manipuliert werden kann, um die dynamische Abstimmung zu erreichen, bedarf weiterer Untersuchungen.
Funding Open Access funding provided by Projekt DEAL.

Open Access Dieser Artikel wird unter der Creative Commons Namensnennung 4.0 International Lizenz veröffentlicht, welche die Nutzung, Vervielfältigung, Bearbeitung, Verbreitung und Wiedergabe in jeglichem Medium und Format erlaubt, sofern Sie den/die ursprünglichen Autor(en) und die Quelle ordnungsgemäß nennen, einen Link zur Creative Commons Lizenz beifügen und angeben, ob Änderungen vorgenommen wurden.

Die in diesem Artikel enthaltenen Bilder und sonstiges Drittmaterial unterliegen ebenfalls der genannten Creative Commons Lizenz, sofern sich aus der Abbildungslegende nichts anderes ergibt. Sofern das betreffende Material nicht unter der genannten Creative Commons Lizenz steht und die betreffende Handlung nicht nach gesetzlichen Vorschriften erlaubt ist, ist für die oben aufgeführten Weiterverwendungen des Materials die Einwilligung des jeweiligen Rechteinhabers einzuholen.

Weitere Details zur Lizenz entnehmen Sie bitte der Lizenzinformation auf http://creativecommons.org/licenses/by/4.0/deed.de.

\section{Literatur}

1. Bauer G (2011) Ölhydraulik. Springer Vieweg, Wiesbaden

2. Beater P (1999) Entwurf hydraulischer Maschinen: Modellbildung, Stabilitätsanalyse und Simulation hydrostatischer Antriebe und Steuerungen. Springer, Heidelberg, Berlin, New York

3. Cundiff JS (2001) Fluid power circuits and controls: fundamentals and applications. CRC Press, Boca Raton

4. Hahn H, Piepenbrink A, Leimbach K (1994) Input/output linearization control of an electro servo-hydraulic actuator. Proc. of the 1994 Conference on Control Applications, S 995-1000

5. Horn J, Bamberger J, Michau P, Pindl S (2003) Flatness-based clutch control for automated manual transmissions. Control Eng Pract 11:1353-1359

6. Jelali M, Kroll A (2003) Hydraulic servo-systems: modelling, identification and control (advances in industrial control). Springer, London

7. Köster MA (2017) On modeling, analysis and nonlinear control of hydraulic systems, Ph.D. thesis, Karlsruher Institut für Technologie (KIT), Karlsruhe

8. Licskó G, Champneys A, Hôs C (2009) Nonlinear Analysis of a Single Stage Pressure Relief Valve. Int J Appl Math 39(4):12-26

9. Maier CC, Schröders S, Ebner W, Köster M, Fidlin A, Hametner C (2019) Modeling and nonlinear parameter identification for hydraulic servo-systems with switching properties. Mechatronics 61:83-95

10. Matthies HJ, Renius KT (2014) Einführung in die Ölhydraulik: Für Studium und Praxis. Springer Vieweg, Wiesbaden

11. Merrit HE (1967) Hydraulic control systems. John Wiley \& Sons Inc., Hoboken

12. Montanari M, Ronchi F, Rossi C, Tilli A, Tonielli A (2004) Control and performance evaluation of a clutch servo system with hydraulic actuation. Control Eng Pract 12:1369-1379

13. Will D, Gebhardt N (2011) Hydraulik: Grundlagen, Komponenten, Schaltungen. Springer, Heidelberg, Berlin, New York 